\title{
Mapping of noise pollution by different interpolation methods in recovery section of Ghandi telecommunication Cables Company
}

\author{
Taghizadeh-Mehrjardi $\mathrm{R}, \mathrm{PhD}^{1^{*}}$, Zare $\mathrm{M}, \mathrm{PhD}^{2}$, Zare S, MSc ${ }^{3}$ \\ 1- Assistant Prof., Faculty of Agriculture and Natural Resources, University of Ardakan, Ardakan, Iran. 2- PhD Student, \\ LUNAM Université, Universitéd'Angers, Laboratoired'Ergonomieetd'Epidémiologie en Santé au Travail (LEEST), \\ Angers, France. 3- MSc, Dept of Electrical Engineering, National Iranian Oil Company (NIOC), Yazd, Iran.
}

\begin{abstract}
Received: September 2013, Accepted: January 2013

Background: Noise pollution and workers' noise exposure are common in industrial factories in Iran. In order to reduce this noise pollution, evaluation and investigation of noise emission are both necessary. In this study, different noise mapping methodsare used for determining the distribution of noise.

Materials and Methods: In the present study, for preparing a noise map in a hall of an industrial factory, sampling was carried out in $6 \times 6 \mathrm{~m}$ grid. After data normalization the variogram was developed. For interpolation of mentioned parameter, kriging and Inverse Distance Weighting methods were used. The best model for interpolation was selected by cross validation and error evaluation methods, such as Route Mean Square Error(RMSE).

Results: The results showed that kriging method is better than other methods for prediction of noise property. The noise map was prepared, using the best interpolation method in Geographical Information System environment.

Conclusion: Workers in this industrial hall were exposed to noise which is mainly induced by noisy machines. Noise maps which were produced in this study showed the distribution of noise and, also revealed that workers suffer from serious noise pollution.
\end{abstract}

Key words: Noise Pollution, Interpolation, Industry.

\section{Introduction}

Noise pollution is a major problem and a complaint among workers within industrial environments (1). It is estimated that most of industries in Iran suffer from problems which are induced by noise (2). Exposure to noise levels, especially higher than threshold limits, will lead to hearing loss and other biological disorders such as cardio vascular effects, sleep disorders and nervous complaints (3). Most of workers within industries in Iran are suffering from noise exposure, and Complaints regarding noise level from workers are high, showing that noise problems are among the important problems in Iran.
In order to manage noise problems in industries, noise distribution must be evaluated and illustrated in work places before proposing any noise control program. Noise map is the best way to represent the sound level distribution existing in a given district.

In general, noise maps have two main purposes. First, they can be used for proposing a noise action plans for management and reduction of noise level. Secondly a noise map is to provide

\footnotetext{
* Corresponding author: Ruhollah TaghizadehMehrjardi, Faculty of Agriculture and Natural Resources, University of Ardakan, Ardakan, Iran. Email: rh_taghizade@yahoo.com
} 
information on noise levels to estimate how many people are affected.

Two main stages can be distinguished in mapping process, 1) the sampling stage, during which measurements are taken from environmental variable at selected locations; and 2) the prediction stage, during which the observations are interpolated to a fine grid. The quality of the resulting map is determined by both stages. Geostatisticians have concentrated most on the second stage, by applying different types of interpolation methods (4).

Geostatistical methods were developed to create mathematical models of spatial correlation structures with a variogram as the quantitative measure of spatial correlation. The variogram is commonly used in geostatistics and the interpolation technique of kriging, providing the "best', unbiased, linear estimate of a regionalized variable in alocation where sampling isn't done, where "best" is defined in a least-squares sense. The emphasis is set on local accuracy, i.e. closeness of the estimate to the actual, but unknown value without any regard for the global statistical properties of the estimates. Kriging estimation variances are independent from the estimated values and are related to the spatial arrangement of the sample data and variogrammodel (5).

The accuracy of different spatial interpolation methods for pollution parameters mapping in soil, air and water have been evaluated by some research recently (6). Kriging method was used to estimate heavy metals concentration in groundwater and concluded that it is the best estimator for spatial prediction of Lead (7). Tsai et al used geographic information systems (GIS) and concluded that noise maps are a useful way of evaluating noise levels (8). The present study aimed to evaluate accuracy of two interpolation methods (kriging and IDW (Inverse Distance Weighting)) for prediction of noise values in Recovery section of Ghandi Telecommunication Cable Co. Noise map of this department also was provided to be compared for the noise level distribution with noise regulation standards.

\section{Materials and Methods}

This is a case study which was done in the recovery section of Ghandi Telecommunication Cable Co. in summer 2011. This section has 66 meter length and 66 meter wide with 7 meter height. The floor and walls were made of concrete and break. 25 people worked in this department and the most important noise resources were Prickly Machine, Caterpillar, Halter Table, Peeler Wiring and Mill. The location of study area and distribution of 106 sampling points (data was collected based on systematic method) is shown in Figure 1.

In this study for spatial prediction of noise exposure, 106 temporary noise monitoring stations in the Recovery Section were selected to determine noise exposure status during eight hour working period. Moreover, during noise measurement (eight hour working period), other factors (i.e. Temperature and air condition) were mostly stable. To identify noisy areas, noise survey was conducted within Recovery Section, and it was divided to $6 \times 6 \mathrm{~m}$ stations. The sample sizes were selected based on the recovery section area. Noise measurement was done in centre of each station at 2 meter height. The measurement was done with a sound level meter model TES 52A on slow response and A-filter. The sound level meter was held at arm's length. The sound level meter had also been calibrated before and after each use. The calibrator model was TES-1356, and 
calibration was done in $1000 \mathrm{~Hz}$ frequency and $94 \mathrm{~dB}$ sound level.

After collection and normalization of data (Logarithmic method), kriging and IDW methods of interpolation were used. Finally, the best method of interpolation was selected using cross-validation. The study was proceeded to prepare the map based on this interpolation and the Geographical Information System. Figure 2 shows the flowchart of this study.

Kriging: The presence of a spatial structure where observations are close to each other are more alike than those that are far apart (spatial autocorrelation) is a prerequisite to the application of geostatistics $(4 ; 9)$. The experimental variogram measures the average degree of dissimilarity between unsampled values and a nearby data value (10; 11 ), and thus can depict autocorrelation at various distances. The value of the experimental variogram for a separation distance of $\mathrm{h}$ (referred to as the lag) is half the average squared difference between the value at $\mathrm{z}(\mathrm{xi})$ and the value at $\mathrm{z}(\mathrm{xi}+\mathrm{h})$ $(9 ; 11)$.

$$
\gamma(h)=\frac{1}{2 \mathrm{n}(\mathrm{h})} \sum_{i=1}^{n(h)}[Z(x i)-Z(x i+h)]^{2}
$$

Where $\mathrm{n}(\mathrm{h})$ is the number of data pairs within a given class of distance and direction. If the values at $\mathrm{Z}(\mathrm{xi})$ and $\mathrm{Z}(\mathrm{xi}+\mathrm{h})$ are auto correlated the result of Eq. (1) will be small, relative to an uncorrelated pair of points. From analysis of the experimental variogram, a suitable model (e.g. spherical, exponential) is then fitted, usually by weighted least squares, and the parameters (e.g. range, nugget and sill) are then used in the kriging procedure.

IDW: In interpolation with IDW method, a weight is attributed to the point to be measured. The amount of this weight is depended to the distance of the point to another unknown point. These weights are controlled on the bases of power of ten. With increase of power of ten, the effect of the points that are farther diminishes. Lesser power distributes the weights more uniformly between neighbouring points.

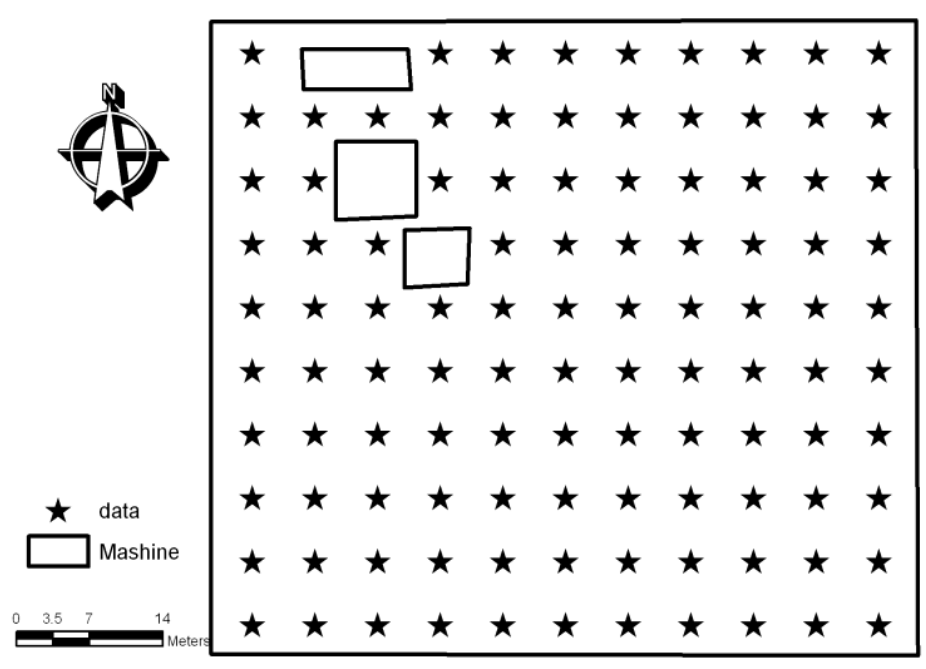

Figure1: Distribution of sampling point in Recovery section of Telecommunication Cable Company 
We should keep in mind that in this method the distance between the points count, so the points of equal distance have equal weights [12].

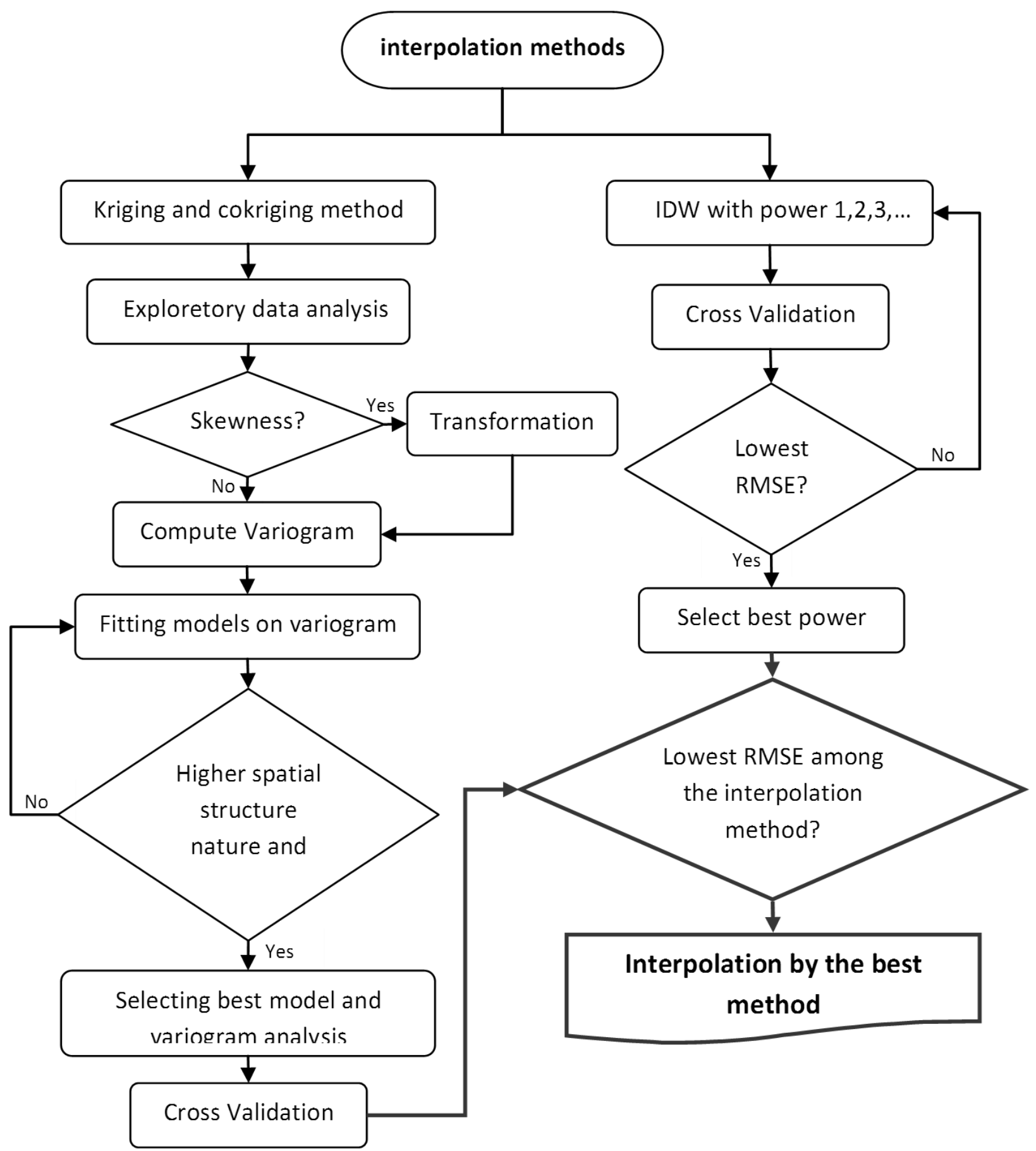

Figure 2: Flowchart of Geostatistic study and selection of the best model for estimation of variable 
In this method the weight factor is calculated with the use of the following formula:

$$
\lambda_{\mathrm{i}}=\frac{D_{i}^{-\alpha}}{\sum_{i=1}^{n} D_{i}^{-\alpha}}
$$

$\lambda_{i}=$ the weight of point, ${ }^{D_{i}}=$ The distance between point $\mathrm{i}$ and the unknown point, $\alpha=$ The power ten of weight

Finally, RMSE was used to evaluate model performances in cross-validation mode (13). The smallest RMSE indicate the most accurate predictions. The RMSE was derived according to Eqs. (4).

R.M.S.E $=\sqrt{\frac{1}{N} \sum_{i=1}^{N}\left(Z\left(x_{i}\right)-Z^{*}\left(x_{i}\right)\right)^{2}}$

$Z(x i)$ is observed value at point $x i, Z^{*}(x i)$ is predicted value at point $x i, N$ is number of samples.

\section{Results}

The variable exhibits a non-normal distribution of measured values and therefore does not initially satisfy the basic assumption of geostatistics of statistical normality. This restriction is eliminated, by applying a data amenable transform to the sample values that make them more to analysis and estimation. The most useful data transform is the logtransform.

Since natural log values can be back transformed to real values, we can use a semi-variogram model derived from the transformed sample values to predict the spatial variation of logarithmic values of the noise. A statistical summary of the measuring noise in various locations near machines is presented in Table 1. As shown in this table, the parameter had high skeness, therefore itwas normalized using logarithmic method (5).

Our task now is to fit models to the experimental or sample values choosing models and fitting them to data remain among the most controversial topics in geostatistic. There are still controversial who fit models by eye and who defined their practice with vigor. They may justify their attitude on the grounds that when kriging the resulting estimates are much the same for all reasonable models of the variogram. There are others who fit models numerically and automatically using "black box" software, often without any choice, judgment or control.

Table 1: statistical analysis of noise value in various locations at Recovery Section

\begin{tabular}{cccccccc}
\hline Measuring location & LP Min & LP Max & LP Mean & SD & Kurtosis & skeness & Exposure Time \\
\hline Near Mill Machine & 89.6 & 91.2 & 90.36 & 0.67 & -0.5 & 0.4 & 141 minutes \\
Prickly Machine & 79.9 & 81.7 & 80.8 & 0.69 & -0.3 & 0.3 & 232 minutes \\
Caterpillar & 79.8 & 81.7 & 80.98 & 0.69 & -0.3 & 0.4 & 21 minutes \\
Halter Table & 79.6 & 81.4 & 80.66 & 0.69 & -0.5 & 0.2 & 95 minutes \\
Peeler Wiring & 81.5 & 84.1 & 82.86 & 1.05 & -0.3 & 0.4 & 220 minutes \\
$\quad$ Noise in all of & 77.9 & 92.1 & 84.54 & 3.28 & -0.4 & 0.3 & 293 minutes \\
Recovery section & & & & &
\end{tabular}


This tool can have unfortunate consequences. We used a procedure that embodies both visual inspection and statistical fitting, as follow. First plot the experimental variogram. Then choose, from the models, one or more with approximately the right shape and with sufficient detail to the principal trends in the experimental values. The first step in using of kriging method is investigating the presence of spatial structure among the data by variogram analysis. For achievement to this issue, variograms was computed using normal data. Variogram related to kriging method is presented in Figure 3. The best model for fitting on experimental variogram was selected based on less RSS values (Table 2). Therefore, it was recognized that Gaussian model is suitable for estimation of noise value.

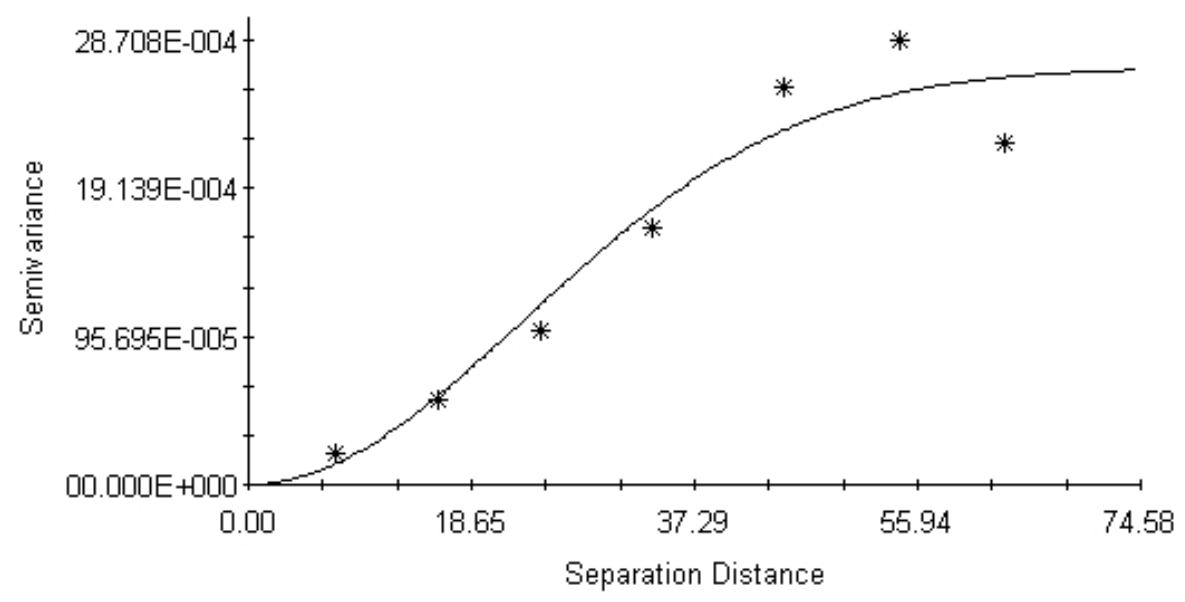

Figure 3: Variogram related to kriging method

Also, table 3 illustrates the parameters of the variogram. The ratio of nugget variance to sill expressed in percentages can be regarded as a criterion for classifying the spatial dependence of parameters. If this ratio is less than $25 \%$, then the variable has strong spatial dependence; if the ratio is between 25 and
$75 \%$, the variable has moderate spatial dependence; and greater than $75 \%$, the variables shows only weak spatial dependence. Since this ratio for all of the noise value is less than $25 \%$, it has strong spatial dependence (14).

Table 2: RSS value of different models of variogram

\begin{tabular}{ccccc}
\hline Model & Gaussian & Spherical & Exponential & Linear \\
\cline { 2 - 5 } RSS & $\mathbf{3 . 7 e - 7}$ & $5.08 \mathrm{e}-7$ & $6.1 \mathrm{e}-7$ & $4.8 \mathrm{e}-7$ \\
\hline
\end{tabular}


Table 3: Best-fitted variogram models and their parameters

\begin{tabular}{ccccc}
\hline Property & Model & Nugget & Sill & Range \\
\cline { 2 - 5 } Noise & Gaussian & $1 \mathrm{e}-16$ & $2.69 \mathrm{e}-16$ & 32.5 \\
\hline
\end{tabular}

For determination of the most suitable method, among Kriging and IDW, RMSE was used. Results showed that geostatistical method had more considerable accuracy than IDW (Table 4). Our results are similar to the findings of Xiaopeng and Lingqing (15). They also reported that geostatistic methods had more considerable accuracy than IDW method for preparing maps.

Table 4: Results of the interpolation error based on RMSE value

\begin{tabular}{lcccccc}
\hline & \multicolumn{1}{c}{ kriging } & \multicolumn{5}{c}{ IDW } \\
Property & & 1 & 2 & 3 & 4 & 5 \\
\cline { 2 - 7 } Noise & 0.97 & 1.02 & 1.03 & 1.04 & 1.07 & 1.09 \\
\cline { 3 - 7 }
\end{tabular}

Result of cross validation illustrated in figure 4. As shown in this graph, accuracy of prediction method is reliable. Finally, a map of noise value was prepared using kriging which was the best method for interpolation in GIS environment (Figure 5).

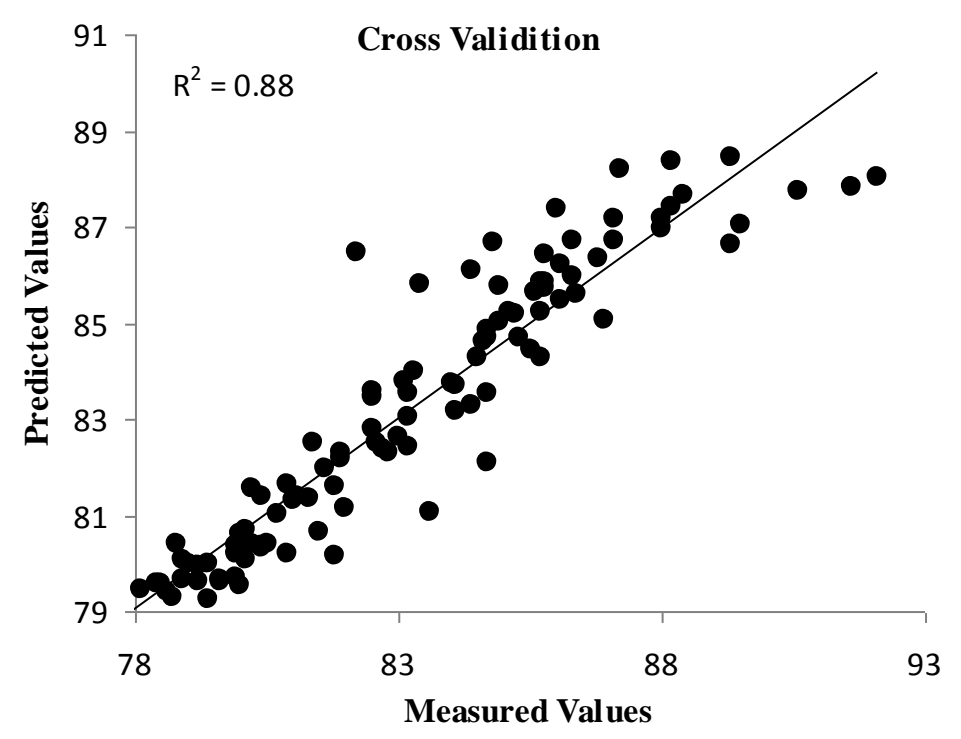

Figure4: Cross-Validation of Kriging method 

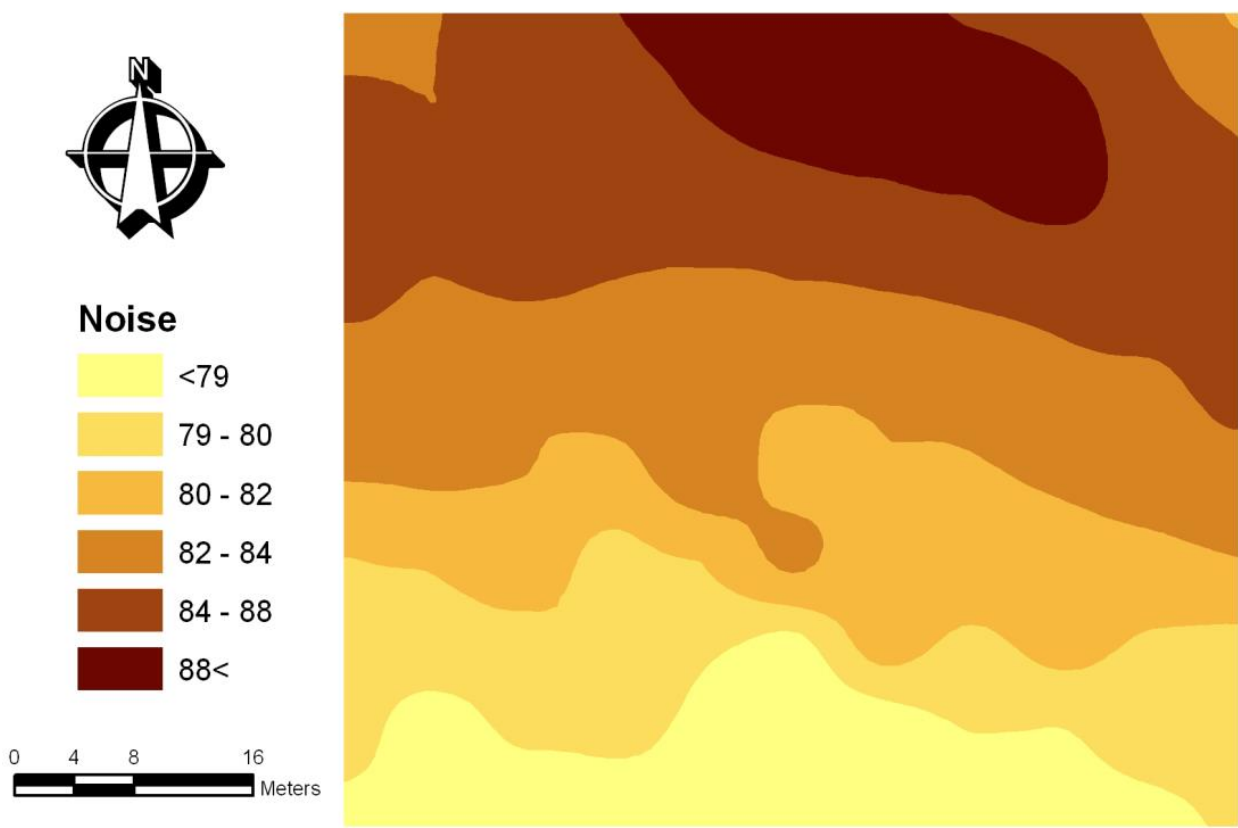

Figure5: The map of spatial distribution of noise value using of kriging method

\section{Discussion}

The noise map (Figure 5) indicates the noise levels for eight hours in Recovery Section, and it shows distributions of noise levels in all of locations in this Section. As shown in this Fig., high noise levels between $84-88 \mathrm{~dB}$ can be observed near operational machines. In this area, there are a number of noise sources such as Prickly Machine, Caterpillar, Halter Table, Peeler Wiring, Mill, vacuum pumps, couch rolls, air and steam valves etc. Each makes a contribution to the overall sound pressure level (noise level) at a given position. But the highest level of noise, $>88 \mathrm{~dB}$, is in the North of the hall where it is not near the major machine in the field, it is probably related to old fans operating in this area. Noise mapping in this study simply showed measurements at predetermined positions identified by applying a $6 \mathrm{~m}$ grid to the floor plan which it has high accuracy than traditional methods.

To indicate the locations with noise level higher than governmental noise regulations, measuring noise levels monitored in the map were compared with the Iranian Noise Standards which was produced by Ministry of Health (Figure6). As shown, the noise level exceeds Noise Standards in some locations where workers were working. Also, it is indicated that noise, especially near some machines, is higher or within standard regulation which is $85 \mathrm{~dB}$ A in Iran.

Generally, analysis showed that kriging performed better than IDW technique in characterizing the spatial variability which is in line with the work done by Rizzo and Mouser (16); Nazari-Zadeh et al (17); and Ahmad (18). They also revealed that geostatistical methods are the best model for interpolation, but we must be careful about it. 


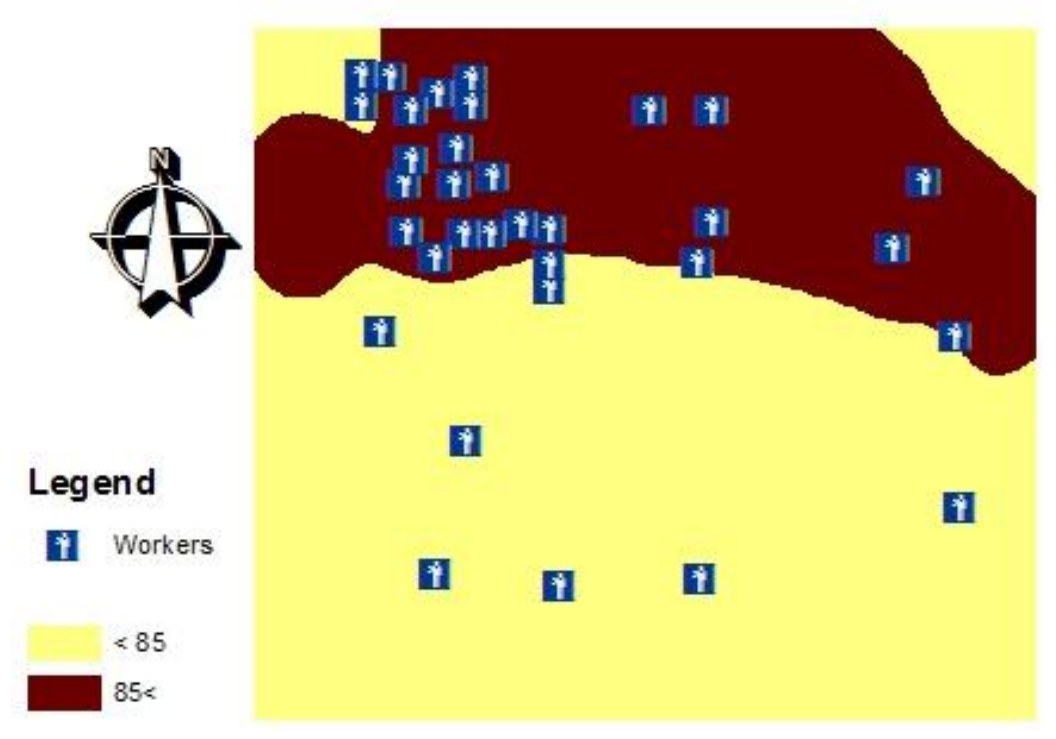

Figure 6: The map of workers distribution exposure to various levels of noise

Geostatistic obviously does not offer a statistical model which is advantageous in every situation. Careful analysis of the measurement data using common sense can sometimes result in the same conclusions as those resulting from lengthily and computationally heavy calculations. In general, as spacing between samples is large compared to the dimensions of the investigated field, the potential advantageous of a geostatisticalanalysis becomes less. For spacing beyond the range of spatial autocorrelation, kriging estimates reduction of the same results as for the classical random sampling. A geostatistical analysis is not only computationally heavy, it requires also an impotent number of samples to be taken and analysed as acute as possible. At least 30 to 50 pairs of observations are necessary to calculate one point of the experimental variogram. Since the lag range over which the variogram is calculated, it should be approximately one fourth to one half of the dimension of the field studied, the experimental variogram should contain points ranging from very small to relative large lags.

As a result, geostatistical investigation is mostly based on hundred, even thousands, of observation. If one observation of the variable is costly, this requirement may jeopardize a geostatistical analysis. Summarized, the disadvantageous of geostatistical approach toward the spatial inventory of soil variables, also called are:

1- In practice, observations need to be numerical.

2- Large data sets are required.

3- Storing information processing power is needed.

The advantages are:

1- It is a reproducible procedure which is easy to verify and update.

2- No classification of data is required. Hence all problems concerning classification disappear. 
3- The numerical output can serve as an input for further processing in GIS

4- It yields as conceptually much more realistic inventory than the traditional groundwater maps.

\section{Conclusion}

Workers in this industrial hall are exposed to noise which is mainly induced by noisy machines. Noise maps which produced in this study showed the distribution of noise and, it revealed that workers suffer from serious noise pollution. Unacceptable noise in which the more percentage of the workers are exposed must be managed by control action urgently. This study showed that a useful way for evaluating and illustrating noise level in industrial halls is noise mapping. Additionally, Results showed that geostatistical method had more considerable accuracy than IDW method. Using this method can identify the locations which immediately requirenoise controls.

\section{Acknowledgements}

The authors would like to acknowledge the University of Ardakan and the recovery section of Ghandi telecommunication Cables Company inYazd province.

Conflict of interests: Non declared.

\section{References}

1. Celik O, Yalcin S, Ozturk A. Hearing parameters in noise exposed industrial workers. Auris Nasus Larynx 1998; 25(4):369-75.

2. Pourabdiyan S, Ghotbi M, Yousefi HA, Habibi E, Zare M. The epidemiologic study on hearing standard threshold shift using audiometric data and noise level among workers of Isfehan metal industry. Koomesh 2009; 10(4):253-60.

3. Abedi K, Zare M, Rahiminezhad M, Valipour E, Barkhordary A, Halvanee $\mathrm{GH}$, et al. Hearing loss among airport employees, Isfahan-Iran. Journal of Gorgan University of Medical Sciences 2010; 11(4):57-63.

4. Goovaerts P. Geostatistics in soil science: state-of-the-art and perspectives. Geoderma 1999; 89(1-2):1-45.

5. Webster R, Oliver MA (2007). Geostatistics for environmental scientists. $2^{\text {nd }}$ ed. Australia, Brisbane: John Wiley \& Sons.

6. Istok JD, Cooper RM. Geostatistics applied to groundwater pollution. III: Global estimates. J Environ Eng 1988; 114(4):91528.

7. D'Agostino V, Greene EA, Passarella G, Vurro M. Spatial and temporal study of nitrate concentration in groundwater by means of coregionalization. Environ Geol 1998; 36(3-4):285-95.

8. Tsai KT, Lin MD, Chen YH. Noise mapping in urban environments: A Taiwan study. Applied Acoustics 2009; 70(7):964-72.

9. Robinson TP, Metternicht G. Testing the performance of spatial interpolation techniques for mapping soil properties. Computers and Electronics in Agriculture 2006; 50(2):97-108.

10. Deutsch CV, Journel AG (1998). GSLIB: Geostatistical software library and user's guide. $2^{\text {nd }}$ ed. New York, Oxford: Oxford University Press. P369.

11. Lark RM. Estimating variograms of soil properties by the method-of-moments and maximum likelihood. Eur J Soil Sci 2000; 51(4):717-28.

12. Burrough PA, McDonnell RA. Creating continuous surfaces from point data. In: Principles of Geographic Information Systems. $2^{\text {nd }}$ ed. Oxford: Oxford University Press. P 98-131.

13. Davis BM. Uses and abuses of crossvalidation in geostatistics. Math Geol 1987; 19(3):241-8.

14. Shi J, Wang H, Xu J, Wu J, Liu X, Zhu H, et al. Spatial distribution of heavy metals in soils: a case study of Changxing, China. Environ Geol 2007; 52(1):1-10.

15. Yang $X$, Wang L. Spatial analysis and hazard assessment of mercury in soil around the coal-fired power plant: a case study from the city of Baoji, China. Environ Geol 2008; 
53(7):1381-8.

16. Nazari-zade F, Arshadiyan F, Zand-vakily K. Study of spatial variability of Groundwater quality of Balarood Plain in Khuzestan province. Proceedings of The first congress of optimized exploitation from water source of Karoon and Zayanderood Plain: Shahrekord: Shahrekord University; 2006; P1236-40.

17. Rizzo DM, Mouser PJ. Evaluation of Geostatistics for Combined Hydrochemistry and Microbial Community Fingerprinting at a Waste Disposal Site. Critical Transitions in Water and Environmental Resources Management; 2000. p1-11.

18. Ahmed S. Groundwater monitoring network design: Application of Geostatistics with a few Case studies from a granitic aquifer in a semiarid region. In: Groundwater Hydrology. Sherif MM, Singh VP, Al-Rashed M, editors. Japan: Tokyo, Balkema; 2002; P37-57. 